\title{
Non-linear HRV Analysis to Quantify the Effects of Intermittent Hypoxia Using an OSA Rat Model
}

\author{
Daniel Romero $^{1}$ and Raimon Jané ${ }^{1,2}$ Senior Member, IEEE
}

\begin{abstract}
In this paper, a non-linear HRV analysis was performed to assess fragmentation signatures observed in heartbeat time series after intermittent hypoxia (IH). Three markers quantifying short-term fragmentation levels, $P I P, I A L S$ and $P S S$, were evaluated on $\mathbf{R}-\mathbf{R}$ interval series obtained in a rat model of recurrent apnea. Through airway obstructions, apnea episodes were periodically simulated in six anesthetized Sprague-Dawley rats. The number of apnea events per hour (AHI index) was varied during the first half of the experiment while apnea episodes lasted $15 \mathrm{~s}$. For the second part, apnea episodes lasted 5, 10 or $15 s$, but the AHI index was fixed. Recurrent apnea was repeated for $15-\mathrm{min}$ time intervals in all cases, alternating with basal periods of the same duration. The fragmentation markers were evaluated in segments of 5 minutes, selected at the beginning and end of the experiment. The impact of the heart and breathing rates (HR and BR, respectively) on the parameter estimates was also investigated. The results obtained show a significant increase (from 5 to $10 \%, p<0.05)$ in fragmentation measures of heartbeat time series after IH, indicating a clear deterioration of the initial conditions. Moreover, there was a strong linear relationship $(r>0.9)$ between these markers and BR, as well as with the ratio given by $H R / B R$. Although fragmentation may be impacted by IH, we found that it is highly dependent on HR and BR values and thus, they should be considered during its calculation or used to normalize the fragmentation estimates.
\end{abstract}

\section{INTRODUCTION}

Obstructive sleep apnea (OSA) is a chronic health condition characterized by repeated episodes of apnea during sleep, resulting in sustained exposure to intermittent hypoxia (IH). This chronic condition has been linked to some cardiovascular consequences of OSA, such as systemic hypertension, myocardial infarction and stroke [1], [2], [3].

To date, the underlying mechanisms linking $\mathrm{IH}$ to vascular diseases in OSA are unclear. However, it has been reported that an elevated sympathetic tone of the autonomic nervous system (ANS) [4], oxidative stress and endothelial dysfunction [2], inflammation and atherosclerosis [3] somehow contribute to this matter.

\footnotetext{
*This work supported in part by CERCA Program, Secretaria dUniversitats i Recerca del Departament dEmpresa i Coneixement de la Generalitat de Catalunya (GRC 2017 SGR 01770) and the Spanish Ministry of Economy and Competitiveness (DPI2015-68820-R MINECO/FEDER). D.R. acknowledges the financial support of the Juan de la Cierva fellowship from MINECO, Ref: IJCI-2016-30696.

${ }^{1}$ Daniel Romero is with the BIOSPIN group at the Institute for Bioengineering of Catalonia (IBEC), the Barcelona Institute of Science and Technology (BIST), Barcelona, Spain. dromero@ibecbarcelona.eu

${ }^{2} \mathrm{R}$. Jané is with the Institute for Bioengineering of Catalonia (IBEC), the Barcelona Institute of Science and Technology (BIST), Centro de Investigación Biomédica en Red de Bioingeniería, Biomateriales y Nanomedicina (CIBER-BBN) and ESAII Department, Universitat Politècnica de Catalunya - BarcelonaTech (UPC), Barcelona, Spain. rjanedibecbarcelona.eu
}

Many research studies in OSA have developed experimental and human models of acute/chronic IH to figure out the association between OSA and cardiovascular diseases. For instance, in animal studies, the rat models represent one of the most common experiments used for understanding and studying physiological mechanisms involved in sleep apnea. However, rats are usually anesthetized, which can affect cardiovascular functions and the control handled by the autonomic nervous system (ANS) could be partially blocked. Although the dose, type and method used for anesthetics administration can disturb in different ways this control system in rats [6], a recent study reported increased sympathetic nerve activity, sympathetic peripheral chemoreflex sensitivity and central sympathetic respiratory coupling after $\mathrm{IH}$ in anesthetized rats [7].

To study the induced-hypoxia effects caused by OSA, a rat model of chronic recurrent airway obstructions was developed [8], [9]. This model was completed [10] with an external control varying the duration and frequency of the induced apneas. Different sensors were included in order to acquire cardiac and respiratory activity, such as flow and pressure transducers, electrodes and a specific pulse oximeter for rat monitoring.

This study aims at characterizing the effects of intermittent hypoxia after completion of several recurrent apnea episodes. Modifications in the ANS activity after OSA episodes were evaluated by means of fragmentation signatures observed in heartbeat time series, analyzed during the start and end of the experiment.

\section{MATERIALS AND METHODS}

\section{A. Experimental data}

Six male Sprague-Dawley rats (mean weight: $437 \pm 27 \mathrm{~g}$ ) were intraperitoneally anesthetized with uretane $(1 \mathrm{~g} / 1 \mathrm{~kg})$. The rats were connected to a system that incorporates a controlled nasal mask, including one tube open to the atmosphere and another connected to a positive pressure pump, preventing the animal from rebreathing. OSA episodes were simulated by obstructing the airways in the tubes using electrovalves. The electrovalves were controlled by software using the Biopac ${ }^{\circledR}$ Systems. The electrodes, nasal mask and $\mathrm{SpO}_{2}$ sensor were placed on the animals after anesthesia and shaving (Fig 1). The experimental procedure involving the animal model described here was approved by the Institutional Animal Care and Ethics Committee.

Several signals were acquired during spontaneous breathing and simulated OSA episodes. Respiratory flow signal, respiratory pressure and electrocardiogram (ECG) signal 
(leads I and II) were registered by Biopac ${ }^{\circledR}$ Systems. Photoplesthysmography signal and $\mathrm{SpO}_{2}$ were measured by a pulse oximeter (Mouse OxPlus ${ }^{\circledR}$ ) positioned in the rat leg.

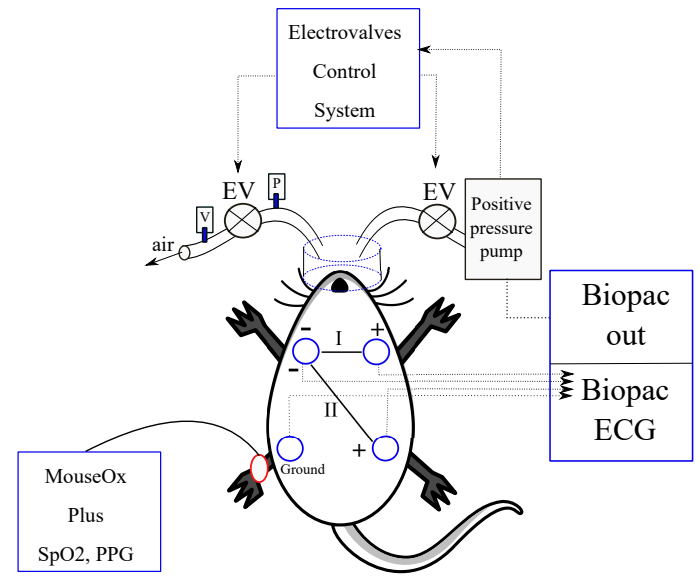

Fig. 1. Experimental setup for the OSA model, using two electrovalves (EV) and a positive pressure pump. Signal acquired: respiratory flow (V') and pressure (P), $\mathrm{SpO}_{2}$ and PPG (MouseOx Plus ${ }^{\circledR}$ ) and ECG ( Biopac ${ }^{\circledR}$ ).

\section{B. Experimental Protocol}

Two different protocols were set for the experiment. In both of them, recurrent apnea episodes were induced for 15 -min intervals followed by 15 -min resting periods. Apnea index (AI) of 20, 40 and 60 apneas/hour were applied in the first protocol with apnea episodes duration of $15 \mathrm{~s}$ (Fig. 2-a). In the second protocol (Fig. 2-b), AI was fixed to 60 and the apnea episodes lasted either 5,10 or $15 \mathrm{~s}$. In both configurations, the order of the 15-min intervals of recurrent apnea was randomly selected. Desaturation levels, assessed from $\mathrm{SpO}_{2}$ signals, ranged between 5-23\% with respect to the pre-apnea values. For this study, only the initial and final basal intervals (end of the second protocol) were considered to evaluate heart rate fragmentation before and after apnea episodes, to assess the cumulative effect of IH.

\section{ECG Preprocessing}

ECG signals, sampled at $1250 \mathrm{~Hz}$, were preprocessed before the automatic extraction of the evaluated marker, including automatic QRS complex detection [11] and visual inspection to remove abnormal beats, baseline drift attenuation, 4-th order bidirectional Butterworth low-pass filtering at $45 \mathrm{~Hz}$ to remove power line interference and high frequency noise.

\section{Non-linear HRV analysis}

After QRS complexes detection, the time positions of consecutive R-wave peaks were determined and used to build the RR interval series in all rats as follows:

$$
R R[i]=R[i+1]-R[i] ; \quad i=1, \ldots N-1
$$

where $i$ and $N$ represent the $i$-th beat and the total number of beats of the analyzed segment. a)

\begin{tabular}{|c|c|c|c|c|c|c|c|}
\hline Rat & Bas 1 & A15s & Bas 2 & A15s & Bas 3 & A15s & Bas 4 \\
\hline 1.1 & & $\mathrm{Al} 20$ & & Al40 & & Al60 & \\
\hline 1.2 & & $\mathrm{Al} 40$ & & $\mathrm{Al} 20$ & & Al60 & \\
\hline 1.3 & & Al60 & & $\mathrm{Al} 20$ & & Al40 & \\
\hline 1.4 & & $\mathrm{Al} 40$ & & Al60 & & $\mathrm{Al} 20$ & \\
\hline 1.6 & & $\mathrm{Al} 20$ & & Al60 & & Al40 & \\
\hline 1.7 & & Al60 & & Al40 & & Al20 & \\
\hline
\end{tabular}

b)

\begin{tabular}{|c|c|c|c|c|c|c|c|}
\hline Rat & Bas 1 & Al60 & Bas 2 & A160 & Bas 3 & Al60 & Bas 4 \\
\hline 1.1 & & $\mathrm{~A} 5 \mathrm{~s}$ & & A10s & & A15s & \\
\hline 1.2 & & A5s & & A15s & & A10s & \\
\hline 1.3 & & A10s & & A5s & & A15s & \\
\hline 1.4 & & $\mathrm{~A} 15 \mathrm{~s}$ & & A10s & & A5s & \\
\hline 1.6 & & A10s & & A15s & & A5s & \\
\hline 1.7 & & A15s & & A5s & & A10s & \\
\hline
\end{tabular}

Fig. 2. a) Protocol 1: Sequences of 15 -s apnea episodes used during 15 -min recurrent apnea intervals, preceded and followed by resting periods of 15 minutes (Bas \#), applied for each rat. b) Protocol 2: Sequences of recurrent apnea episodes (with $\mathrm{AI}=60$ ) for 15 -min intervals, preceded and followed by resting resting period of 15 minutes (Bas \#).

To assess the level of fragmentation present in $R R$ interval series, a set of recently proposed markers were evaluated on normal-to-normal $(N N)$ interval series, obtained by considering only normal sinus heartbeats [5]. Abnormal intervals were replaced by the average of the previous 5 intervals. The fragmentation indices are the following:

- PIP: The percentage of inflection points in the $N N$ time series, that is, the number of zero-crossing points in the first derivative of the $N N$ series referred to their total lengths.

- IALS: The inverse average length of acceleration/deceleration segments. Acceleration/deceleration segments represent consecutive $N N$ intervals between adjacent inflection points, where the difference between 2 consecutive $N N$ intervals is negative or positive, respectively.

- PSS: The percentage of $N N$ intervals that are in short segments. Short segments are defined as those acceleration or deceleration segments that lasted $\geq 3 \mathrm{NN}$ intervals.

According to the definition of fragmentation, the above indices will be higher if a time series is more fragmented. Importantly, removing the presence of non-sinus heartbeats from the annotation step is crucial because it can increase fragmentation in the analyzed time series [5]. Nevertheless, since these parameters can be highly influenced by both the respiratory rate (BR) and heart rate (HR), we also investigated their relationships during the experiment. 


\section{E. Statistical analysis}

Results obtained are expressed in mean \pm SD. The Wilcoxon signed rank nonparametric test was applied to compare paired observations obtained at different stages of the experiment. The relationships of the parameters with HR and BR was determine using the Pearson's correlation coefficient. The significance level was set to 0.05 in all cases.

\section{RESULTS}

The Fig. 3 shows an example of two $N N$ segments (initial and final), analyzed in one rat of the study population. It can be observed that the initial segment is more regular than the final one. Moreover, the HR is quite different in both segments, being higher $(>20 \%)$ during the initial stage. Respiratory rate (BR) is slightly lower $(\sim 17 \%)$ and the number of inflections points (IP) is smaller at the end, however, the PIP value is higher. This suggests that some kind of relationship may exist between fragmentation and HR, BR, or a combination of both.
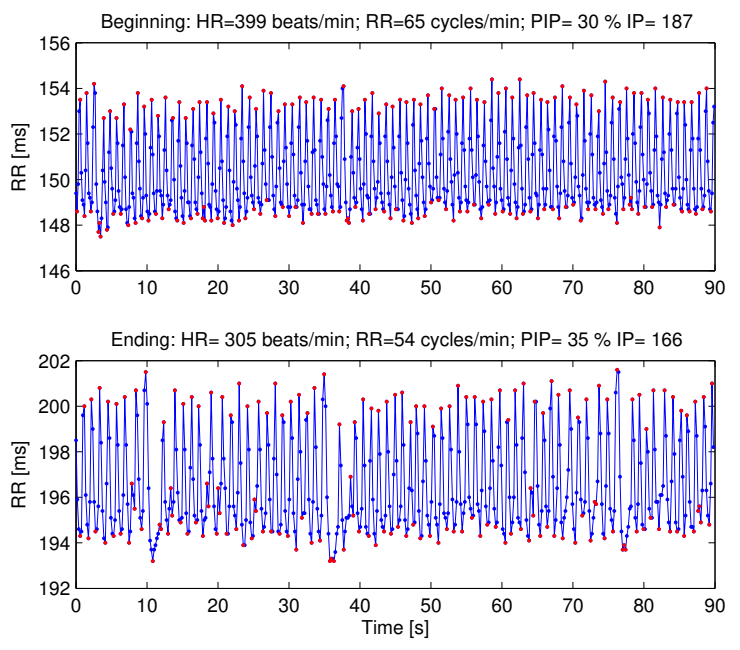

Fig. 3. $\quad N N$ time series representing 90-s segments during the initial and final stage of the experiment. Red points indicate inflection points detected on the NN series. Heart rate, respiratory rate, PIP value and the number of IP are displayed in the top of each graph.

Table 1 summarizes the results obtained for all markers and rats before and after IH. It can be observed that both the HR and HR/BR significantly decreased by the end of the experiment while the PIP and PSS markers were significantly higher, indicating an increase in fragmentation of the time series analyzed. The $p$-values obtained show that only BR did not change significantly between both time periods.

In Fig. 4 are plotted the PIP values against the HR, BR and the ratio HR/BR for each animal, evaluated during the initial and final stages. The straight lines represent the model obtained after fitting a linear function to all data. From the figure, we can observe that $\mathrm{BR}$ and the HR/BR ratio have a strong linear relationship with the PIP values when the experiment started. However, in the case of HR, some animals with similar values (see Fig. 4-(a)) present very different fragmentation measures in the same period, meaning that BR
TABLE I

MEAN \pm SD OF THE NON-LINEAR HRV PARAMETERS, HEART RATE AND RESPIRATORY RATE.

\begin{tabular}{|c||c|c|c|}
\hline Parameters & Before IH & After IH & $p$-value \\
\hline HR (beats/min) & $385 \pm 26$ & $350 \pm 29$ & 0.0156 \\
BR (cycles/min) & $92 \pm 18$ & $90 \pm 19$ & 0.2813 \\
HR/BR (beats/cycles) & $4.3 \pm 1.0$ & $4.0 \pm 0.8$ & 0.0469 \\
PIP (\%) & $46.5 \pm 7.8$ & $50.3 \pm 5.5$ & 0.0313 \\
PSS (\%) & $64.7 \pm 27.0$ & $74.8 \pm 24.1$ & 0.0469 \\
IALS (\%) & $0.46 \pm 0.08$ & $0.51 \pm 0.05$ & 0.0313 \\
\hline
\end{tabular}

seems to have a stronger impact on fragmentation estimates as compared to HR.
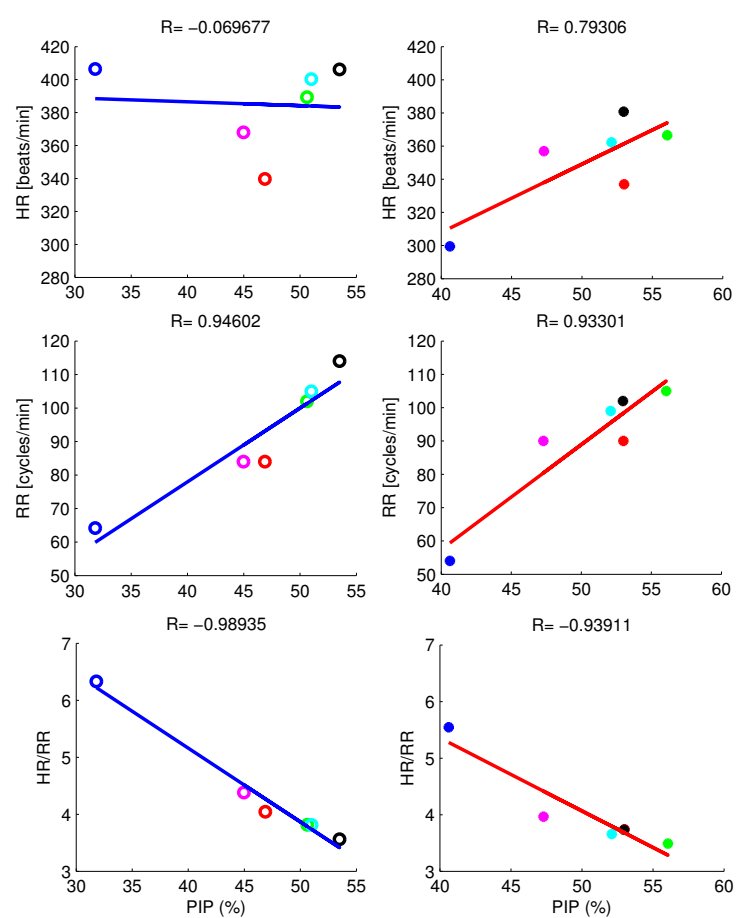

Fig. 4. PIP values vs HR, RR and HR/RR ratio obtained for all animals before (left column) and after (right column) the IH. R stands for Pearson's correlation coefficient.

The same analysis was performed after completion of the recurrent apnea episodes to assess their effects. Results are displayed in the right column of Fig. 4. In most rats, HR and BR decreased with respect to the initial values, confirming the results of Table I. The HR/BR ratio was also lower at the final stage, keeping in some extent their linear relationship with the fragmentation values. The major difference was observed in the analysis of HR vs PIP, where the linear model fitted better to the actual data as compared to the initial ones. Similar results were also obtained for the PSS and IALS markers, which are summarized in Table II.

In addition to the actual values, the changes developed during the experiment by the analyzed markers were also investigated. Fig. 5 presents the correlation values obtained when analyzing the changes seen in fragmentation markers vs those changes occurred in $\mathrm{HR}, \mathrm{BR}$ an their ratio. In 

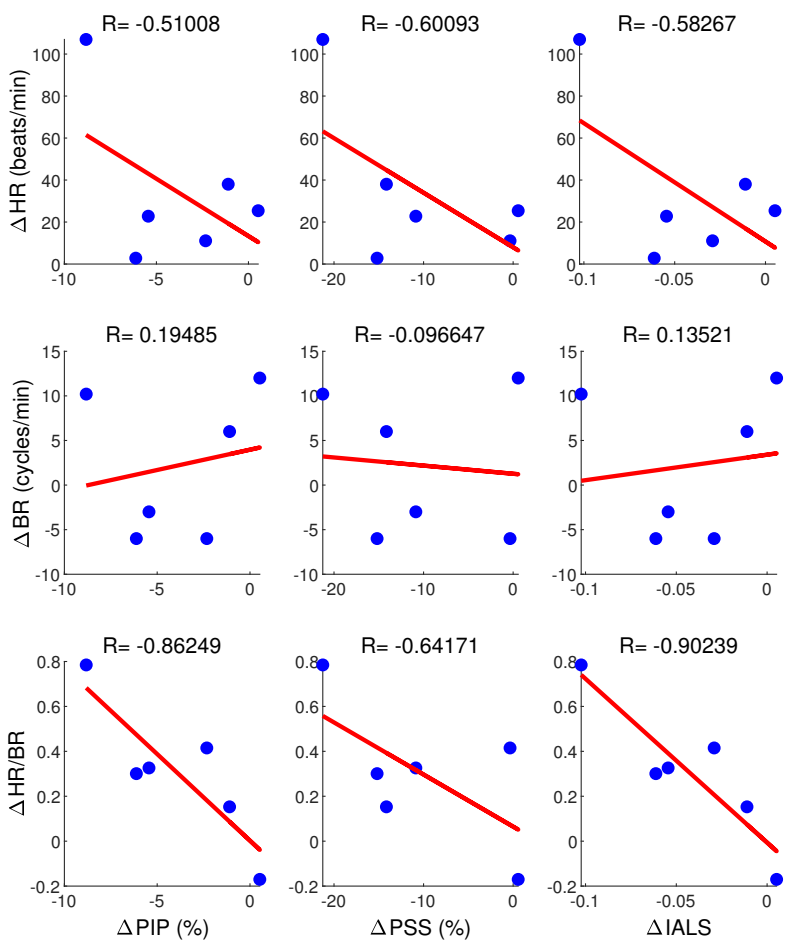

Fig. 5. Correlation values obtained between changes observed in fragmentation markers and in heart rate, respiratory rates and their ratio.

this analysis, only HR/BR seems to have a stronger linear relationship with all of them.

TABLE II

CORRELATION VALUES OBTAINED BETWEEN FRAGMENTATION MARKERS AND THE HEART AND RESPIRATORY RATES.

\begin{tabular}{|c||c|c|c|c|c|}
\hline \multicolumn{1}{|c||}{} & \multicolumn{2}{c|}{ HR } & \multicolumn{2}{c|}{ BR } & \multicolumn{2}{c|}{ HR/BR } \\
Parameters & Before $\mid$ After & \multicolumn{2}{c|}{ Before | After } & Before | After \\
\hline PIP (\%) & $-0.07 \mid 0.79$ & $0.95 \mid 0.93$ & $-0.99 \mid-0.94$ \\
PSS (\%) & $-0.07 \mid 0.72$ & $0.94 \mid 0.85$ & $-0.97 \mid-0.85$ \\
IALS (\%) & $-0.07 \mid 0.79$ & $0.95 \mid 0.93$ & $-0.99 \mid-0.93$ \\
\hline
\end{tabular}

\section{DISCUSSION AND CONCLUSIONS}

In this study, we evaluated the level of fragmentation observed in heartbeat time series and how they are affected by intermittent hypoxia. To performed this analysis, we evaluated three fragmentation indices on RR interval time series, extracted from the initial and final periods of an OSA model in rats. The results obtained shown that the level of fragmentation significantly increased (between $5 \%$ and $10 \%$, $p<0.05$ ) after completion of several sequences of recurrent apnea episodes, which caused intermittent hypoxia during the whole experiment.

A important finding of the study is related to the fact that both the respiratory rate and its relationship to heart rate are highly correlated with fragmentation parameters $(|R|>0.9)$. Comparable results were also obtained when HR/BR changes were correlated with these parameters. This finding suggests that these variables could be used to obtain indirect measures of fragmentation levels, or at least should be considered during their calculation.

Costa et al. [5] reported that fragmentation of heartbeat time series increases with aging in healthy subjects as well as in patients with heart diseases such as coronary artery disease (CAD). However, fragmentation tends to be higher in patients with CAD than in healthy subjects of similar ages. They also showed that fragmentation seems not to be affected by the mean heart rate, however, our study showed a high correlation with respiratory rate and the ratio between HR and BR, even under different conditions. Nevertheless, our results should be interpreted with caution due to the small size of the population.

It is known that intermittent hypoxia caused by obstructive sleep apnea may contribute to impaired autonomic cardiac function. These alterations are mainly driven by the sympathetic activation and may represent mechanisms for increased occurrence of cardiac arrhythmias in OSA patients. We concluded that the investigated parameters could be useful to assess how much the HRV is affected after recurrent hypoxia episodes, and they should be analyzed and compared to other traditional HRV markers to determine their added value in patients with OSA.

\section{ACKNOWLEDGMENT}

The authors are grateful to Ramon Farré, Daniel Navajas, Josep Maria Montserrat, Isaac Almendros, Marta Torres and Puy Ruiz for their excellent help in designing and implementing the animal model.

\section{REFERENCES}

[1] N.R. Prabhakar. Invited Review: Oxygen sensing during intermittent hypoxia: cellular and molecular mechanisms. J Appl Physiol, vol. 90, pp. 1986-1994, 2001.

[2] L. Lavie. Obstructive sleep apnoea syndrome - an oxidative stress disorder. Sleep Med Rev, vol. 7, pp. 35-51, 2003.

[3] L. Lavie. Sleep-disordered breathing and cerebrovascular disease: a mechanistic approach. Neurol Clin, vol. 23, pp. 1059-1075, 2005.

[4] E.C. Fletcher. Invited review: Physiological consequences of intermittent hypoxia: systemic blood pressure. J Appl Physiol, vol. 90, pp. 1600-1605, 2001.

[5] M.D. Costa, R.B. Davis, A.L. Goldberger. Heart rate fragmentation: a new approach to the analysis of cardiac interbeat interval dynamics. Front Physiol, vol 8, pp. 255. 2017.

[6] A. Shimokawa, T. Kunitake, M. Takasaki, H. Kannan. Differential effects of anesthetics on sympathetic nerve activity and arterial baroreceptor reflex in chronically instrumented rats. J. Auton. Nerv. Syst., vol. 72, pp. 46-54, 1998.

[7] S.J. Kim, A.Y. Fong, P.M. Pilowsky, S.B. Abbott. Sympathoexcitation following intermittent hypoxia in rat is mediated by circulating angiotensin II acting at the carotid body and subfornical organ. J. Physiol, vol. 596, pp. 3217-3232, 2018.

[8] Farré, R., Ncher, M., Serrano-Mollar, A., Gldiz, J. B., Alvarez, F. J., Navajas, D., Montserrat, J. M. Rat model of chronic recurrent airway obstructions to study the sleep apnea syndrome. Sleep, vol. 30, pp. 930-933, 2007.

[9] A. Carreras, I. Almendros, I. Acerbi, J.M. Montserrat, D. Navajas, R. Farr. Obstructive apneas induce early release of mesenchymal stem cells into circulating blood. Sleep, vol. 32, pp. 117-119, 2009.

[10] R. Jané R, J. Lázaro, P. Ruiz, E. Gil, D. Navajas, R. Farré, P. Laguna. Obstructive Sleep Apnea in a rat model: Effects of anesthesia on autonomic evaluation from heart rate variability measures. In proc. Computing in Cardiology (CinC), pp. 1011-1014, 2013.

[11] Martínez, J. P., Almeida, R., Olmos, S., Rocha, A. P., Laguna, P. A wavelet-based ECG delineator: evaluation on standard databases. IEEE Trans. Biomed. Eng, vol. 51, pp. 570-581, 2004. 